\title{
Erratum
}

Anaesthesist $2021 \cdot 70: 548$

https://doi.org/10.1007/s00101-021-00981-0

Online publiziert: 2. Juni 2021

(C) Der/die Autor(en) 2021

Mark Michael ${ }^{1}$. Noemi Freise ${ }^{2} \cdot$ Verena Keitel $^{2} \cdot$ Andreas Schaper $^{3}$. Christian Plettenberg ${ }^{4} \cdot$ Sven Dreyer ${ }^{5} \cdot$ Michael Bernhard $^{1}$

'Zentrale Notaufnahme, Universitätsklinikum Düsseldorf, Düsseldorf, Deutschland

${ }^{2}$ Klinik für Gastroenterologie, Hepatologie und Infektiologie, Universitätsklinikum Düsseldorf, Düsseldorf, Deutschland

${ }^{3}$ Giftinformationszentrum-Nord, Universitätsmedizin Göttingen, Göttingen, Deutschland

${ }^{4}$ Klinik für Hals-Nasen-Ohrenheilkunde, Universitätsklinikum Düsseldorf, Düsseldorf, Deutschland

${ }^{5}$ Hyperbare Sauerstofftherapie, Universitätsklinikum Düsseldorf, Düsseldorf, Deutschland

\section{Erratum zu: Akzidentelle Atemkalkingestion im Rahmen eines Tauchganges}

\section{Erratum zu:}

Anaesthesist 2021

https://doi.org/10.1007/s00101-021-

00920-z

In der Zusammenfassung des zuerst publizierten Artikels wurde die Zusammensetzung des Atemkalks nicht korrekt dargestellt. Bestandteile des Atemkalks sind Kalziumhydroxid und Natronlauge $(\mathrm{NaOH})$. Hydroxycarbamid ist ein zytostatisch wirkendes Arzneimittel.

Der vollständige und korrigierten Artikel steht Ihnen auf www.springermedi zin.de (• Abb. 1) zur Verfügung. Bitte geben Sie dort den Beitragstitel in die Suche ein.

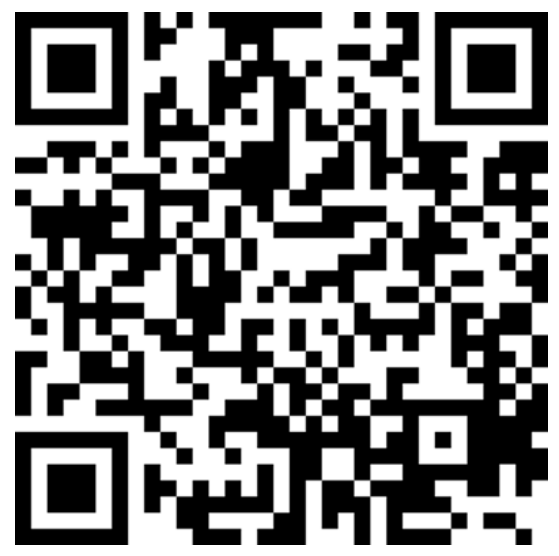

Abb. $1 \Delta$ QR-Code zum korrigierten Artikel

\section{Korrespondenzadresse}

Dr. med. Mark Michael

Zentrale Notaufnahme, Universitätsklinikum Düsseldorf

Moorenstraße 5, 40225 Düsseldorf,

Deutschland

Mark.Michael@med.uni-duesseldorf.de
Die Online-Version des Originalartikels ist unter https://doi.org/10.1007/s00101-021-00920-z zu finden. 